Article

\title{
The Industrial Organism Corynebacterium glutamicum Requires Mycothiol as Antioxidant to Resist Against Oxidative Stress in Bioreactor Cultivations
}

\author{
Fabian Stefan Franz Hartmann ${ }^{1}$, Lina Clermont ${ }^{2}$, Quach Ngoc Tung ${ }^{3,+}{ }^{+}$, Haike Antelmann ${ }^{3}(\mathbb{D}$ \\ and Gerd Michael Seibold $1,2, * \mathbb{D}$ \\ 1 Section for Synthetic Biology, Department of Biotechnology and Biomedicine, \\ Technical University of Denmark, 2800 Kongens Lyngby, Denmark; fashart@dtu.dk \\ 2 Institute of Biochemistry, University of Cologne, 51149 Cologne, Germany; linaclermont@gmx.de \\ 3 Institute of Biology-Microbiology, Freie Universität Berlin, 10115-14199 Berlin, Germany; \\ qtung@zedat.fu-berlin.de (Q.N.T.); haike.antelmann@fu-berlin.de (H.A.) \\ * Correspondence: gesei@dtu.dk \\ + Current address: Institute of Biotechnology, Vietnam Academy of Science and Technology, \\ Hanoi 10000, Vietnam.
}

Received: 11 September 2020; Accepted: 29 September 2020; Published: 9 October 2020

\begin{abstract}
In aerobic environments, bacteria are exposed to reactive oxygen species (ROS). To avoid an excess of ROS, microorganisms are equipped with powerful enzymatic and non-enzymatic antioxidants. Corynebacterium glutamicum, a widely used industrial platform organism, uses mycothiol (MSH) as major low molecular weight (LMW) thiol and non-enzymatic antioxidant. In aerobic bioreactor cultivations, C. glutamicum becomes exposed to oxygen concentrations surpassing the air saturation, which are supposed to constitute a challenge for the intracellular MSH redox balance. In this study, the role of MSH was investigated at different oxygen levels $\left(\mathrm{pO}_{2}\right)$ in bioreactor cultivations in C. glutamicum. Despite the presence of other highly efficient antioxidant systems, such as catalase, the MSH deficient $\triangle m s h C$ mutant was impaired in growth in bioreactor experiments performed at $\mathrm{pO}_{2}$ values of $30 \%$. At a $\mathrm{pO}_{2}$ level of $20 \%$, this growth defect was abolished, indicating a high susceptibility of the MSH-deficient mutant towards elevated oxygen concentrations. Bioreactor experiments with C. glutamicum expressing the Mrx1-roGFP2 redox biosensor revealed a strong oxidative shift in the $\mathrm{MSH}$ redox potential $\left(E_{\mathrm{MSH}}\right)$ at $\mathrm{pO}_{2}$ values above $20 \%$. This indicates that the LMW thiol MSH is an essential antioxidant to maintain the robustness and industrial performance of C. glutamicum during aerobic fermentation processes.
\end{abstract}

Keywords: Corynebacterium glutamicum; oxidative stress; mycothiol; Mrx1-roGFP2; redox potential

\section{Introduction}

The Gram-positive soil bacterium Corynebacterium glutamicum is widely used as anindustrial workhorse primarily for the production of L-glutamate and L-lysine [1], and has been genetically engineered as a broad platform for production of several important industrial products [2,3]. Currently, C. glutamiucm is mostly used for aerobic production processes, but its facultative anaerobic metabolism allows to design efficient two-stage processes for the production of reduced chemicals including an aerobic growth phase and an anaerobic production phase [4,5].

Reactive oxygen species (ROS) are generated as an inescapable consequence of aerobic metabolism, caused by incomplete stepwise reduction of molecular oxygen during respiration $[6,7]$. ROS include superoxide anions $\left(\mathrm{O}_{2}^{-}\right)$, hydroxyl radicals $\left({ }^{\bullet} \mathrm{OH}\right)$, peroxy radicals $\left(\mathrm{ROO}^{\bullet}\right)$, alkoxy radicals $\left(\mathrm{RO}^{\bullet}\right)$, and hydrogen peroxide $\left(\mathrm{H}_{2} \mathrm{O}_{2}\right)$ [6-10]. An excess of ROS leads to oxidative stress, which subsequently 
damages essential cell components, such as lipids, proteins, and nucleic acids [11]. To avoid irreversible damages, aerobic living organisms possess a wide range of ROS-scavengers including enzymatic and non-enzymatic detoxification systems [12-17].

Non-enzymatic antioxidant defense systems are represented by low molecular weight (LMW) thiols, which are essential to maintain a reducing environment in the cytoplasm [16]. Eukaryotes and most Gram-negative bacteria produce glutathione (GSH) as their major LMW thiol [18]. Bacteria of the Gram-positive phylum Actinobacteria, including Mycobacterium tuberculosis, Mycobacterium smegmatis, and C. glutamicum, however, utilize the GSH surrogate mycothiol (MSH) [19-22]. Upon the formation of ROS, the redox-active sulfhydryl group of MSH can either scavenge free radicals directly or function as a cofactor for antioxidant enzymes, resulting in the formation of oxidized mycothiol disulfide (MSSM) $[14,15,23,24]$. MSH can prevent overoxidation of protein thiols to sulfonic acids by forming mixed disulfides via a mechanism referred to as protein $S$-mycothiolation [25]. Irreversible overoxidation could cause a loss of cell viability and the requirement of the synthesis of new proteins in the case of essential and abundant proteins $[25,26]$. Upon treatment with hypochlorite, 25 S-mycothiolated proteins have been identified in C. glutamicum, indicating its protective function during oxidative stress [27]. Consequently, the absence of MSH was shown to increase oxidative stress sensitivity indicated by an oxidized environment, an impaired growth behavior, and a loss of cell viability [27-29].

Previous research regarding the physiological role of antioxidants in C. glutamicum exclusively used artificial oxidants to induce ROS generation rather than using conditions more relevant for production conditions such as bioreactor cultivations at different aeration rates [12,15,27-29]. Of note, ROS production rates are proportional to the collision frequency of oxygen and redox enzymes [7-9,30-32]. Especially during aerobic industrial fermentations, cells are exposed to oxygen concentrations often surpassing the air saturation. Hyperbaric oxygen was shown to be detrimental for growth patterns of various organisms, for example, Escherichia coli, Bacillus subtilis, and Saccharomyces cerevisiae [33-35]. C. glutamicum, as an aerobic industrial platform organism, is highly robust towards oscillations of low and high oxygen concentrations [36]. However, to the best of our knowledge, the contribution of the non-enzymatic antioxidant and main LMW thiol MSH in C. glutamicum to its robustness during aerobic fermentations has yet not been investigated.

Here, we studied the relevance of the non-enzymatic antioxidant MSH in the industrial platform bacterium C. glutamicum during aerobic batch fermentations. Bioreactor experiments revealed an impaired growth behavior in the MSH deficient C. glutamicum $\triangle m s h C$ mutant upon exposure to oxygen concentrations surpassing air saturation. Application of $C$. glutamicum strains expressing the stably integrated Mrx1-roGFP2 redox biosensor [29] enabled monitoring of the changes in the MSH redox potential $\left(E_{\mathrm{MSH}}\right)$ in C. glutamicum WT during bioreactor cultivations at different oxygen levels. Altogether, the results of our study demonstrate the physiological importance of MSH as a non-enzymatic antioxidant in C. glutamicum to overcome oxidative stress during aerobic bioreactor cultivations.

\section{Materials and Methods}

\subsection{Strains, Media, and Culture Conditions}

The strains used in this study were C. glutamicum ATCC13032 (WT) [37], the MSH-deficient C. glutamicum $\triangle m s h C$ deletion mutant [26], and the Mrx1-roGFP2 redox biosensor expressing strains C. glutamicum WT_Mrx1-roGFP2 and C.glutamicum $\triangle m$ shC_Mrx1-roGFP2 [29]. C. glutamicum strains were pre-cultured in $2 x T Y$ medium at $30{ }^{\circ} \mathrm{C}$ in $500 \mathrm{~mL}$ shake flasks. Prior to inoculation of the main-culture, cells of an overnight culture were washed twice with $100 \mathrm{mM}$ potassium phosphate buffer (pH 7.0). C. glutamicum main cultures were grown in CGXII minimal medium [1] supplemented with $10 \mathrm{~g} \mathrm{~L}^{-1}$ or $20 \mathrm{~g} \mathrm{~L}^{-1}$ glucose as a carbon source for growth experiments in shake flasks and bioreactors, respectively. 
Growth experiments in bioreactors were performed aerobically at $30^{\circ} \mathrm{C}$ as $1 \mathrm{~L}$ cultures in $1.5 \mathrm{~L}$ jars in a BIOSTAT ${ }^{\circledR}$ B fermentation system (Sartorius, Goettingen, Lower Saxony, Germany), as described previously [38]. The $\mathrm{pH}$ was maintained at 7.0 by online measurement using a standard $\mathrm{pH}$ electrode (Mettler Toledo, Giessen, Hessen, Germany) and addition of $4 \mathrm{M} \mathrm{KOH}$ and $4 \mathrm{M} \mathrm{H}_{2} \mathrm{SO}_{4}$. Partial oxygen pressure $\left(\mathrm{pO}_{2}\right)$ was measured online using a polarimetric oxygen electrode (Mettler Toledo), and was adjusted to $\mathrm{pO}_{2}$ values provided in the text in a cascade by stirring at 200 to $800 \mathrm{rpm}$ as well as by mixing $\mathrm{N}_{2}$ and air for the inlet gas. For anaerobic condition $\left(\mathrm{pO}_{2}=0 \%\right), 100 \% \mathrm{~N}_{2}$ was used until a $\mathrm{pO}_{2}$ of $0 \%$ was reached. When required, AF204 antifoam agent (Sigma, MI, USA.) was added manually. The data were collected with the software MFCS (Sartorius BBI Systems, Goettingen, Lower Saxony, Germany). Growth in shake flasks and bioreactors was followed by measuring the optical density (OD 600 $\mathrm{nm}$ ).

\subsection{Fluorescence Measurements of Mrx1-roGFP2 Biosensor Oxidation In Vitro and In Vivo}

For testing suitable settings for fluorescence measurements of Mrx1-roGFP2 redox biosensor oxidation, C. glutamicum strains harboring genomic integrated Mrx1-roGFP2 [29] were pre-cultured in 2xTY medium until the stationary phase. For preparation of crude cell extracts, cells were harvested by centrifugation (4000 rpm, $\left.10 \mathrm{~min} ., 4^{\circ} \mathrm{C}\right)$, washed twice in potassium phosphate buffer $(100 \mathrm{mM}, \mathrm{pH} 7.0)$, and finally resuspended in $1 \mathrm{~mL}$ of the respective buffer solution. Disruption of the cells was conducted using a Ribolyzer (Precellys TM Control Device, Bertin Technologies, Montigny-le-Bretonneux, Department Yvelines, France) at $6000 \mathrm{rpm}$, four times for $30 \mathrm{~s}$ each. Cell debris were removed by centrifugation $\left(12,000 \mathrm{rpm}, 20 \mathrm{~min} ; 4^{\circ} \mathrm{C}\right)$ and $180 \mu \mathrm{L}$ of the supernatant was transferred to black, flat-bottomed 96-well microplates (Thermo Fisher Scientific, Dreieich, Hessen, Germany) for further fluorescence analysis using a fluorescence spectrophotometer (SpectraMax iD3, Molecular Devices LLC, San Jose, CA, USA). After the addition of $20 \mu \mathrm{L}$ oxidants (50 mM diamide), reductants (100 mM Dithiothreitol (DTT), and $100 \mathrm{mM}$ potassium phosphate buffer for fully oxidized, fully reduced, and non-treated control samples, respectively, cells were incubated for $15 \mathrm{~min}$ at $30{ }^{\circ} \mathrm{C}$ as described previously [29]. Subsequently, excitation scans were conducted ( $360 \mathrm{~nm}-470 \mathrm{~nm}$ ) by setting an emission wavelength of $510 \mathrm{~nm}$. For in vivo fluorescence measurements, C. glutamicum strains expressing the Mrx1-roGFP2 biosensor were harvested by centrifugation (4000 rpm, $4 \mathrm{~min}$ ) and washed in $100 \mathrm{mM}$ potassium phosphate buffer ( $\mathrm{pH} 7.0$ ). Finally, an optical density of 40 was adjusted and $180 \mu \mathrm{L}$ of the cell suspension was transferred to black, flat-bottomed 96-well microplates for fluorescence analysis. To determine the maximum and minimum oxidation ratios, $20 \mu \mathrm{L}$ of different concentrated cumene hydroperoxide (CHP) and DTT solutions were added for oxidation and reduction of the biosensor probe, respectively, until the respective ratio reached its minimum and maximum value. For samples, $20 \mu \mathrm{L}$ potassium phosphate buffer was added instead. Mrx1-roGFP2 fluorescence intensity was recorded at an emission intensity of $510 \mathrm{~nm}$ upon excitation at $380 \mathrm{~nm}$ and $470 \mathrm{~nm}$. The corresponding biosensor oxidation degree $(\mathrm{OxD})$ was calculated by normalizing to fully reduced as well as oxidized controls, as described previously [29,39,40], with the following Equation (1):

$$
\mathrm{OxD}=\frac{I 380_{\text {sample }} \times I 470_{\text {red }}-I 380_{\text {red }} \times I 470_{\text {sample }}}{I 380_{\text {sample }} \times I 470_{\text {red }}-I 380_{\text {sample }} \times I 470_{\text {ox }}+I 380_{\text {ox }} \times I 470_{\text {sample }}-I 380_{\text {red }} \times I 470_{\text {sample }}}
$$

Here, $1380_{\text {sample }}$ and $1470_{\text {sample }}$ represent the measured fluorescence intensities received for an excitation at $380 \mathrm{~nm}$ and $470 \mathrm{~nm}$, respectively. Fully reduced and oxidized controls are given by $1380_{\text {red }}, 1470_{\text {red }}$ and $I 380_{\text {ox }}, I 470_{\text {ox }}$, respectively. Calculated OxD values; the standard midpoint redox potential of roGFP2 $\left(E_{\text {roGFP2 }}^{o^{\prime}}=-280 \mathrm{mV}\right)$ [41]; Faraday constant (F: 96,485 $\left.\mathrm{C} \mathrm{mol}^{-1}\right)$ of electric charge per mole of electrons, where 2 refers to the number of electrons transferred during the redox reaction; the respective 
temperature in Kelvin (T: $303.15 \mathrm{~K}$ ); and the universal gas constant (R: $8.314 \mathrm{~J} \mathrm{~K}^{-1} \mathrm{~mol}^{-1}$ ) were used in order to determine the MSH redox potential $\left(E_{\mathrm{MSH}}\right)$ via the Nernst Equation (2):

$$
E_{\mathrm{MSH}}=E_{\mathrm{roGFP} 2}=E_{\mathrm{roGFP} 2}^{o^{\prime}}-\left(\frac{\mathrm{RT}}{2 \mathrm{~F}}\right) \times \operatorname{In}\left(\frac{1-\mathrm{OxD}}{\mathrm{OxD}}\right)
$$

\subsection{Statistical Analysis}

Analysis of one-way variance (ANOVA) with Tukey's test was used to assess differences of biosensor oxidation degrees derived from C. glutamicum WT and MSH-deficient mutant strains harboring the genetically encoded biosensor Mrx1-roGFP2. Differences were considered statistically significant when $p<0.01$.

\section{Results}

\subsection{The MSH-Deficient Mutant is Susceptible to Elevated Oxygen Concentrations}

To compare growth of $C$. glutamicum WT and the $\Delta m s h C$ mutant, batch cultivations in stirred bioreactors were performed at a $\mathrm{pO}_{2}$ value of $\geq 30 \%$ (regulated in cascade via the stirring rate) and a constant $\mathrm{pH}$ of 7.0. These are common conditions used for production and physiological studies with C. glutamicum [42-45]. Growth of C. glutamicum WT proceeded with a rate of $0.37 \mathrm{~h}^{-1}$ and the cells reached a final $\mathrm{OD}_{600}$ of 29 after $24 \mathrm{~h}$ of cultivation (Figure 1a). Growth of the MSH-deficient $\triangle m s h C$ mutant proceeded slower than C. glutamicum WT within the first hours of the bioreactor cultivation, resulting in a cessation of growth after $4 \mathrm{~h}$ (Figure $1 \mathrm{~b}$ ). During the course of cultivation with C. glutamicum WT, a stable $\mathrm{pO}_{2}$ value of $30 \%$ was reached after an expected initial phase with a higher $\mathrm{pO}_{2}$ (Figure 1a). For the MSH deficient strain, however, the decreased oxygen demand resulted in $\mathrm{pO}_{2}$ values always above the minimal $\mathrm{pO}_{2}$ of $30 \%$ (Figure $1 \mathrm{~b}$ ). This decreased oxygen demand of the $\triangle m s h C$ mutant can be explained by the impaired growth during the first $4 \mathrm{~h}$ of cultivation. The rise of $\mathrm{pO}_{2}$ after $4 \mathrm{~h}$ of cultivation coincidences with the stop of growth of the MSH-deficient mutant (Figure 1b). Moreover, strong foam formation was observed at this phase of the bioreactor cultivation with the $\triangle m s h C$ mutant. Taken together, the growth deficit of the $\Delta m s h C$ mutant revealed its susceptibility towards elevated oxygen concentrations present already during the initial phase of the bioreactor cultivation, when a control strategy was chosen to keep $\mathrm{pO}_{2}$ values $\geq 30 \%$.

To avoid high $\mathrm{pO}_{2}$ values during the initial phase of the cultivations, a different strategy for $\mathrm{pO}_{2}$ control was tested for the $\triangle m s h C$ mutant, thanks to its susceptibility for elevated $\mathrm{pO}_{2}$ levels. By mixing air with nitrogen, $\mathrm{pO}_{2}$ in the bioreactor was adjusted to different levels at an initially constant stirring rate of $400 \mathrm{rpm}$. The results showed that, at a $\mathrm{pO}_{2}$ of max $20 \%$, growth of the $\Delta m s h C$ mutant proceeded with slightly reduced growth rate of $0.31 \mathrm{~h}^{-1}$ (Figure 1d) when compared with a growth rate of $0.37 \mathrm{~h}^{-1}$ (Figure 1c) for the WT. Moreover, at a $\mathrm{pO}_{2}$ of max $20 \%$, C. glutamicum WT and $\Delta m s h C$ mutant cells reached final optical densities of 48 and 41 , respectively (Figure 1c,d).

To test for negative effects of elevated $\mathrm{pO}_{2}$ on growing cultures in bioreactors, C. glutamicum WT and the $\triangle m s h C$ mutant were cultivated initially at a $\mathrm{pO}_{2}$ of $\max 20 \%$ for $3 \mathrm{~h}$ until optical densities of 7 and 6, respectively (Figure 1e,f). Subsequently, the $\mathrm{pO}_{2}$ was increased in a single step to $40 \%$. Whereas growth of $C$. glutamicum WT continued after the increase of $\mathrm{pO}_{2}$ to a final OD of 28 in the course of cultivation (Figure 1e), growth of the $\triangle m s h C$ mutant immediately stopped, resulting in a final OD of 9 (Figure 1f). These experiments showed that the MSH-deficient $\triangle m s h C$ mutant is highly sensitive to $\mathrm{pO}_{2}$ levels above $20 \%$. As MSH protects the cells against oxidative stress, these results indicate that, already at slightly increased $\mathrm{pO}_{2}$ levels, oxidative stress occurs during bioreactor cultivation. 

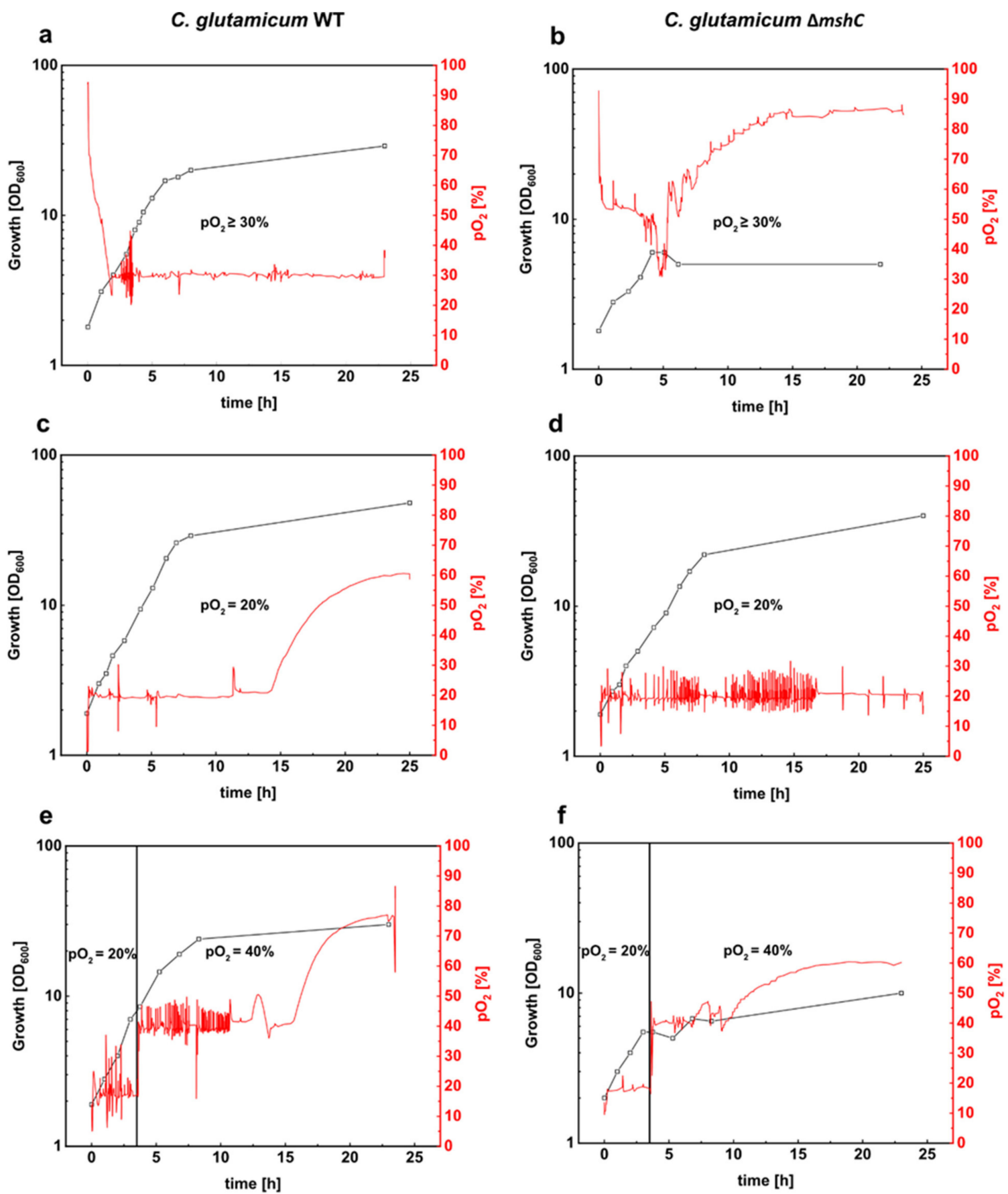

Figure 1. Batch cultivations of C. glutamicum WT (left plots) and the mycothiol-deficient C. glutamicum mutant $\triangle m s h C$ (right plots) in stirred bioreactors. Both strains were cultivated in $1 \mathrm{~L} \mathrm{CgXII} \mathrm{minimal}$ medium $\left(\mathrm{T}=30^{\circ} \mathrm{C}, \mathrm{pH}=7.0\right.$; initial glucose concentration $\left.20 \mathrm{~g} \mathrm{~L}^{-1}\right)$. Bioreactor experiments were performed by setting different $\mathrm{pO}_{2}$ values. $\mathrm{pO}_{2}$ values of $\geq 30 \%$ regulated via stirring ( $200 \mathrm{rpm}-800 \mathrm{rpm}$ ) $(\mathbf{a}, \mathbf{b}) \cdot \mathrm{pO}_{2}$ values of $20 \%$ were set by stirring as well as mixing nitrogen and air for the inlet gas (c,d). Finally, bioreactor experiments were carried out with an initial $\mathrm{pO}_{2}$ value of $20 \%$ during the first $3 \mathrm{~h}$ and a second fermentation phase with a $\mathrm{pO}_{2}$ value of $40 \%(\mathbf{e}, \mathbf{f})$. Growth was monitored by measuring the optical density at $600 \mathrm{~nm}$. Fermentations were performed in BIOSTAT ${ }^{\circledR}$ B bioreactors. Data were collected with the software MFCS. OD, optical density. 


\subsection{Oxidation of the Mrx1-roGFP2 Biosensor Allows Monitoring the Changes in the MSH Redox Potential} $\left(E_{M S H}\right)$ in C. glutamicum

The observation that the MSH deficient $\Delta m s h C$ mutant was impaired in growth with elevated oxygen concentrations prompted us to measure the changes in the MSH redox potential $\left(E_{\mathrm{MSH}}\right)$ in C. glutamicum during bioreactor experiments. Thus, we applied the recently developed genetically encoded Mrx1-roGFP2 biosensor, which is stably integrated in the genome of C. glutamicum [29]. Redox sensitive GFP2 (roGFP2) harbors two Cys residues that form a disulfide bond upon oxidation, resulting in ratiometric changes of two excitation maxima in the fluorescence excitation spectrum [46]. Mrx1 further was shown to selectively reduce S-mycothiolated proteins as part of the Mrx1/MSH/Mtr electron pathway [19,47]. Moreover, the Mrx1-roGFP2 fusion was well characterized as a redox biosensor with respect to its selectivity towards MSSM in vitro [29]. Upon reaction with MSSM, the MSH moiety is transferred to Mrx1 and roGFP2, followed by intramolecular disulfide formation in roGFP2 and the concomitant change of its fluorescence excitation maxima [29] (Figure 2a). To define suitable settings for ratiometric fluorescence measurements, crude cell extracts of C. glutamicum WT_Mrx1-roGFP2 with integrated Mrx1-roGFP2 were prepared and treated with 10 mM DTT or $5 \mathrm{mM}$ diamide for fully reduced and oxidized controls, respectively, as previously described [29]. The strongest fluorescence intensity alteration (emission intensity at $510 \mathrm{~nm}$ ) under our settings was detected when the biosensor was excited at $380 \mathrm{~nm}$ and $470 \mathrm{~nm}$ (Figure S1). More specifically, upon oxidation of the probe, the excitation maximum at $380 \mathrm{~nm}$ increases with the subsequent decrease of the $470 \mathrm{~nm}$ excitation maximum, and vice versa upon reduction of the probe. Although the second fluorescence intensity maximum was described at $488 \mathrm{~nm}$ previously [29], this was out of the range of measurements of our available microplate reader. Thus, we used the calculation of the $380 / 470 \mathrm{~nm}$ fluorescence intensity ratios in our biosensor settings, which is an indicator of the MSH redox potential changes in C. glutamicum.

For in vivo determination of Mrx1-roGFP2 biosensor oxidation, C. glutamicum WT_Mrx1-roGFP2 and $\triangle m s h C \_M r x 1$-roGFP2, both harboring the genome integrated biosensor Mrx1-roGFP2, were cultivated in shake flasks until the stationary phase was reached. Prior to fluorescence measurements, cells were harvested by centrifugation, washed twice with potassium phosphate buffer (100 mM; $\mathrm{pH} 7.0)$, and an optical density of 40 was adjusted as previously described [29]. Upon treatment with DTT and CHP, for reduction and oxidation of the biosensor probe, respectively, the 380/470 nm excitation ratio of Mrx1-roGFP2 was determined (Figure 2b). For non-treated samples, an equal volume of the respective buffer was added instead. Non-treated shake flask samples of C. glutamicum WT_Mrx1-roGFP2 and $\triangle m s h C \_M r x 1-r o G F P 2$ revealed huge differences in terms of the biosensor oxidation ratio with $1.0 \pm 0.02$ and $1.52 \pm 0.03$, respectively (Figure $2 b$ ). However, the addition of DTT (reducing agent) or CHP (oxidizing agent) eliminated the biosensor ratio differences, resulting in fully oxidized and fully reduced biosensor ratios of 1.5-1.6 and 0.7-0.8, respectively (Figure 2b). As expected, growth of the mutant strain in shake flasks with minimal medium proceeded similar with a growth rate of $0.26 \pm 0.02 \mathrm{~h}^{-1}$ when compared with the parental strain C.glutamicum WT_Mrx1-roGFP2 $\left(0.27 \pm 0.03 \mathrm{~h}^{-1}\right)$ (Figure 2c). Biosensor measurements at the end of the exponential growth phase further revealed that the initial biosensor oxidation degrees (OxD; Equation (1)) were maintained highly oxidized (0.91 \pm 0.01 ; $0.86 \pm 0.05)$ in the MSH-deficient mutant and more reduced $(0.6 \pm 0.04 ; 0.49 \pm 0.04)$ in the WT strain (Figure 2d).

This confirms a mycothiol redox potential $\left(E_{\mathrm{MSH}}\right)($ Equation $(2))$ in C.glutamicum WT_Mrx1-roGFP2 and $\triangle m s h C_{-}$Mrx1-roGFP2 of $-280 \pm 2 \mathrm{mV}$ and $-255 \pm 7 \mathrm{mV}$ at the end of the exponential growth phase in shake flasks, respectively (Table 1). This observation is in accordance with the previous study, showing that C. glutamicum WT_Mrx1-roGFP2 maintains a highly reducing intracellular environment during the course of cultivation in shake flasks (-280-300 mV) [29]. In contrast, the MSH-deficient mutant showed a more oxidized intracellular environment [29]. It is likely that elevated ROS levels in the MSH mutant caused an oxidation of Mrx1-roGFP2. However, in contrast to bioreactor experiments with oxygen concentrations surpassing the air saturation $\left(\mathrm{pO}_{2}=30 \%\right)$, growth of the MSH-deficient 
mutant was not impaired in shake flasks (Figure 2c), as seen for bioreactor cultivations with $\mathrm{pO}_{2}$ of $20 \%$ (Figure 1d), when compared with the WT strain. This indicates that ROS production under these conditions did not overwhelm ROS detoxification by MSH-independent enzymatic antioxidant systems in the $\triangle m s h C$ mutant. The addition of the thiol-reactive oxidant $\mathrm{NaOCl}$ to shake flask cultures of the $\triangle m s h C$ mutant was shown to be detrimental in terms of growth patterns [27], as observed during bioreactor experiments with oxygen concentrations surpassing the air saturation. This indicates the sensitivity of the $\triangle m s h C$ mutant towards increased ROS production in bioreactors, supporting the role of MSH to overcome oxidative stress during fermentation. In contrast, MSH is not essential in aerobic shake flask cultures with lower oxygen tension, which is in agreement with the observed more reduced biosensor signals in the C. glutamicum_Mrx1-roGFP2 strain (Figure 2d) [29].

a
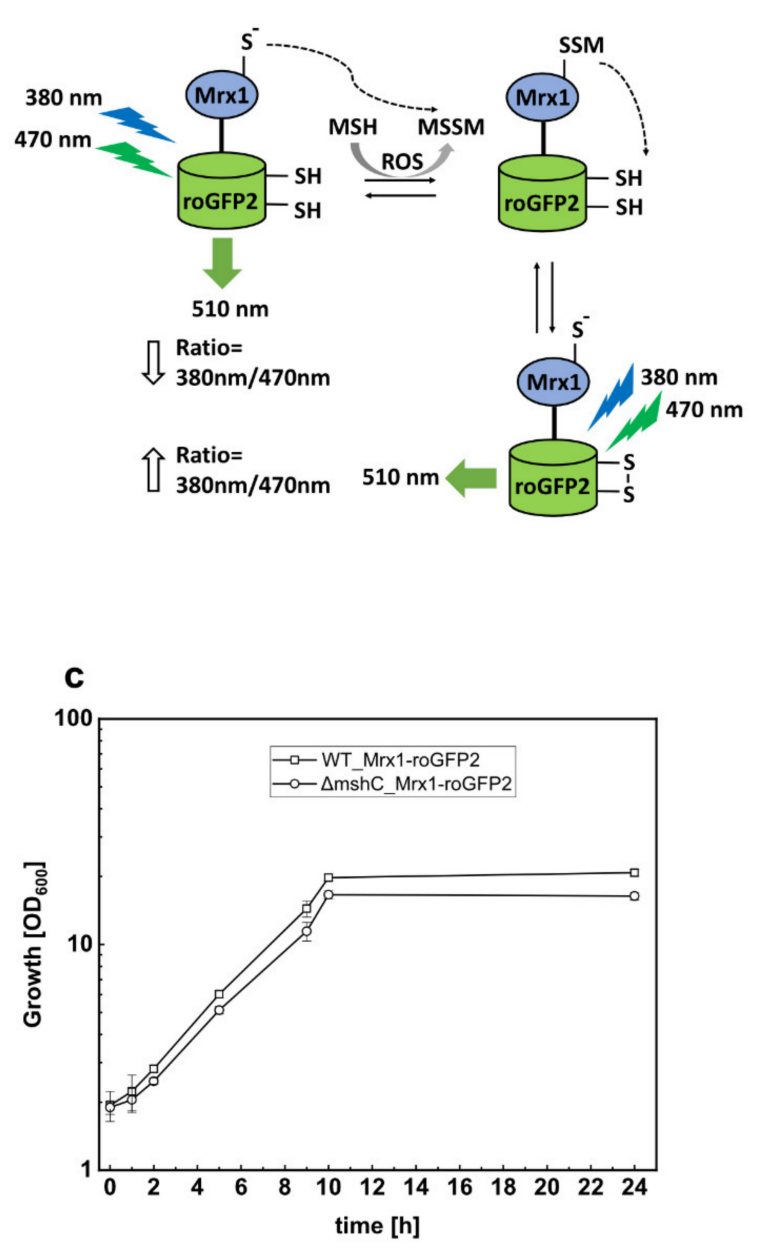

b

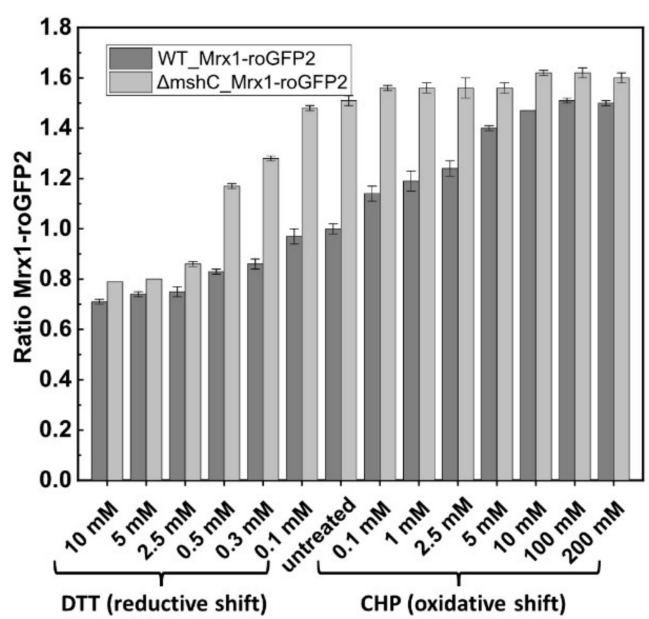

d

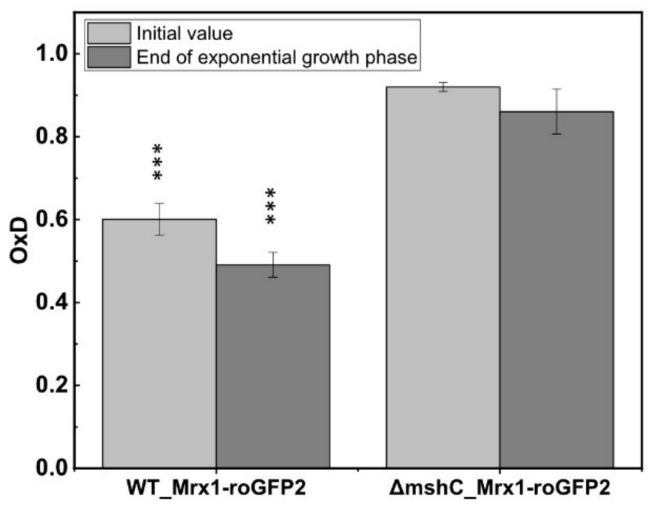

Figure 2. Schematic illustration of the Mrx1-roGFP2 biosensor response mechanism (a); the ratiometric Mrx1-roGFP2 biosensor response as shown by the 380/470 $\mathrm{nm}$ excitation ratio upon treatment with different concentrations of reductants (DTT) and oxidants (CHP) in C. glutamicum WT_Mrx1-roGFP2 (WT_Mrx1-roGFP2) and C. glutamicum $\Delta m s h C \_M r x 1-r o G F P 2$ ( $\left.\Delta m s h C \_M r x 1-r o G F P 2\right)(\mathbf{b})$; shake flasks cultivations of WT_Mrx1-roGFP2 and $\Delta m s h C \_M r x 1-r o G F P 2 ~ i n ~ 50 \mathrm{~mL}$ CGXII minimal medium $\left(\mathrm{T}=30^{\circ} \mathrm{C}\right.$, initial glucose concentration $\left.15 \mathrm{~g} \mathrm{~L}^{-1}, 150 \mathrm{rpm}\right)(\mathrm{c})$; and biosensor oxidation degrees $(\mathrm{OxD})$ derived from shake flask samples after inoculation and the end of exponential growth phase (d). Error bars indicate standard deviations from three independent experiments. OxD values were calculated by normalizing the samples to fully oxidized and reduced controls. OxD values of WT_Mrx1-roGFP2 are significantly different when compared with $\Delta m s h C \_$Mrx1-roGFP2 OxD values at the $p=0.01$ level (one-way analysis of variance (ANOVA) with Tukey's test) ( ${ }^{* * *} p<0.0001$ ). MSH, mycothiol; ROS, reactive oxygen species; MSSM, mycothiol disulfide. 
Table 1. Mycothiol (MSH) redox potential ( $E_{\mathrm{MSH}}$; Nernst equation) during shake flask cultivations.

\begin{tabular}{ccc}
\hline \multirow{2}{*}{ Shake Flask } & \multicolumn{2}{c}{$E_{\mathbf{M S H}}(\mathbf{m V})$} \\
\cline { 2 - 3 } & WT_Mrx1-roGFP2 ${ }^{(a)}$ & $\Delta$ mshC_Mrx1-roGFP2 ${ }^{(b)}$ \\
\hline Initial value & $-274 \pm 2$ & $-248 \pm 2$ \\
End of exponential growth phase & $-280 \pm 2$ & $-255 \pm 7$ \\
\hline lutamicum WT harboring the redox biosensor Mrx1-roGFP2. ${ }^{(\text {b) }}$ MSH-deficient mutant of C. glutamicum \\
ing the redox biosensor Mrx1-roGFP2.
\end{tabular}

(a) C. glutamicum WT harboring the redox bise
harboring the redox biosensor Mrx1-roGFP2.

\subsection{Mycothiol-Dependent Protection is Important when C. glutamicum is Exposed to Elevated} Oxygen Concentrations

To investigate the oxidative response of the Mrx1-roGFP2 biosensor in C. glutamicum WT_Mrx1-roGFP2 at elevated oxygen concentrations, we performed bioreactor experiments with C. glutamicum WT_Mrx1-roGFP2 at a $\mathrm{pO}_{2}$ of $\geq 30 \%$. The first fluorescence measurement revealed an almost fully oxidized biosensor.

To ensure that the biosensor response resulted from increased oxygen concentrations, the bioreactor cultivation of $C$. glutamicum WT_Mrx1-roGFP2 was performed with a stepwise $\mathrm{pO}_{2}$ gradient (Figure 3a). At the initial $\mathrm{pO}_{2}$ of $0 \%$ (set by providing $100 \% \mathrm{~N}_{2}$ as sole gas), the biosensor oxidation ratio was very low, indicating the presence of a reducing environment in the bioreactor at a $\mathrm{pO}_{2}$ of $0 \%$ (Figure $3 \mathrm{a}$ ). Subsequently, the $\mathrm{pO}_{2}$ value was increased in $5 \%$ steps in the bioreactor and, at each of the $\mathrm{pO}_{2}$ steps, the signal ratio of the biosensor was determined $60 \mathrm{~min}$ after setting the $\mathrm{pO}_{2}$. As depicted in Figure $3 a$, the ratio of the biosensor increased when setting higher oxygen concentrations $\left(\mathrm{pO}_{2}\right)$, indicating an oxidative stress response. At a $\mathrm{pO}_{2}$ of $30 \%$, the biosensor oxidation degree (OxD) was determined as $0.86 \pm 0.04$ (Figure $3 \mathrm{~b}$ ), representing a highly oxidized environment in C. glutamicum WT under these conditions. Moreover, further increase of the $\mathrm{pO}_{2}$ value did not lead to enhanced $\mathrm{OxD}$ values, which are not significantly different to those determined for the MSH- deficient mutant strain (Figure $3 b)$. In comparison, at lower $\mathrm{pO}_{2}$ values $\left(\mathrm{pO}_{2}=5 \% ; \mathrm{pO}_{2}=20 \% ; \mathrm{pO}_{2}=25 \%\right), \mathrm{OxD}$ values determined for the WT_Mrx1-roGFP2 strain were significantly lower than the fully oxidized biosensor probes for the $\Delta m s h C \_M r x 1-r o G F P 2$ mutant strain (Figure 3b).

Notably, under aerobic conditions, the strongest oxidative shift occurred when surpassing the air saturation of $20 \%$, resulting in an OxD shift of 0.33 from $0.53 \pm 0.06\left(\mathrm{pO}_{2}=20 \%\right)$ (which is in the range of $\mathrm{OxD}$ values determined for shake flask samples) towards highly oxidized values of $0.86 \pm 0.04$ $\left(\mathrm{pO}_{2}=30 \%\right.$ ) (Figure $\left.3 \mathrm{~b}\right)$. This conforms an oxidative shift of $E_{\mathrm{MSH}}$ from $-280 \pm 6 \mathrm{mV}\left(\mathrm{pO}_{2}=20 \%\right)$ to $-256 \pm 4 \mathrm{mV}\left(\mathrm{pO}_{2}=30 \%\right)$ for the $C$. glutamicum WT_Mrx1-roGFP2 strain, whereas the redox potential of the mutant strain was highly oxidized at every tested $\mathrm{pO}_{2}$ level (Table 2). This oxidative shift is in agreement with the growth defect of the MSH-deficient mutant strain when surpassing the air saturation both when setting a constant $\mathrm{pO}_{2}=30 \%$ (Figure 1d), but also with a stepwise increase of the $\mathrm{pO}_{2}$ value for the mutant strain harboring the redox biosensor Mrx1-roGFP2 (Figure S2).

Taken together, the strong oxidative shift of C. glutamicum WT_Mrx1-roGFP2 in bioreactor cultivations indicates the requirement of the non-enzymatic antioxidant and LMW thiol MSH to overcome oxidative stress when the oxygen concentration surpasses the air saturation.

Table 2. Mycothiol redox potential ( $E_{\mathrm{MSH}}$; Nernst equation) during bioreactor cultivations.

\begin{tabular}{ccc}
\hline \multirow{2}{*}{$\begin{array}{c}\text { Bioreactor } \\
\mathbf{p O}_{2} \text { level (\%) }\end{array}$} & \multicolumn{2}{c}{$E_{\text {MSH }}(\mathbf{m V})$} \\
\cline { 2 - 3 } & WT_Mrx1-roGFP2 $^{\text {(a) }}$ & $\Delta_{\text {mshC_Mrx1-roGFP2 }}{ }^{\text {b) }}$ \\
\hline 5 & $-287 \pm 4$ & $-204 \pm 2$ \\
20 & $-280 \pm 6$ & $-191 \pm 2$ \\
25 & $-272 \pm 3$ & $-208 \pm 22$ \\
$30 \%$ & $-256 \pm 4$ & $-242 \pm 7$ \\
$\geq 30 \%$ & $-246 \pm 28$ & $-218 \pm 23$
\end{tabular}

(a) C. glutamicum WT harboring the redox biosensor Mrx1-roGFP2. ${ }^{(b)}$ MSH-deficient mutant of C. glutamicum harboring the redox biosensor Mrx1-roGFP2. 
a

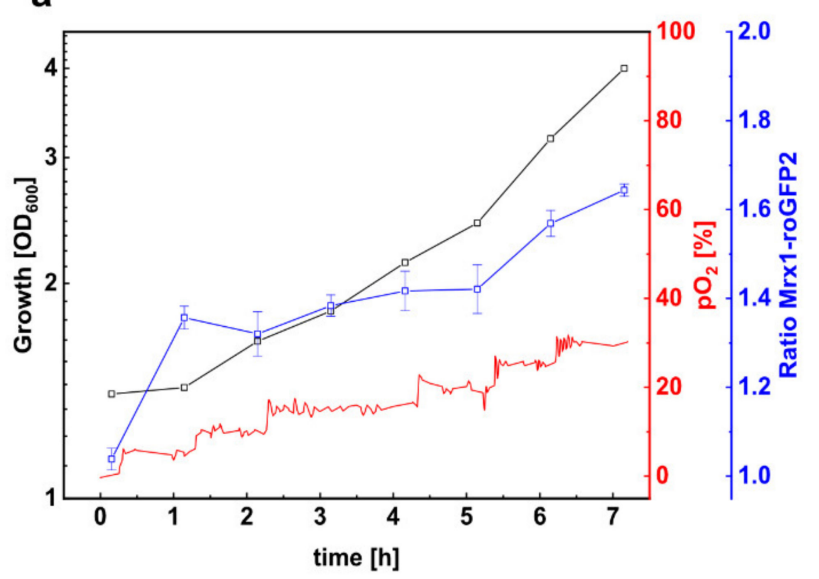

b

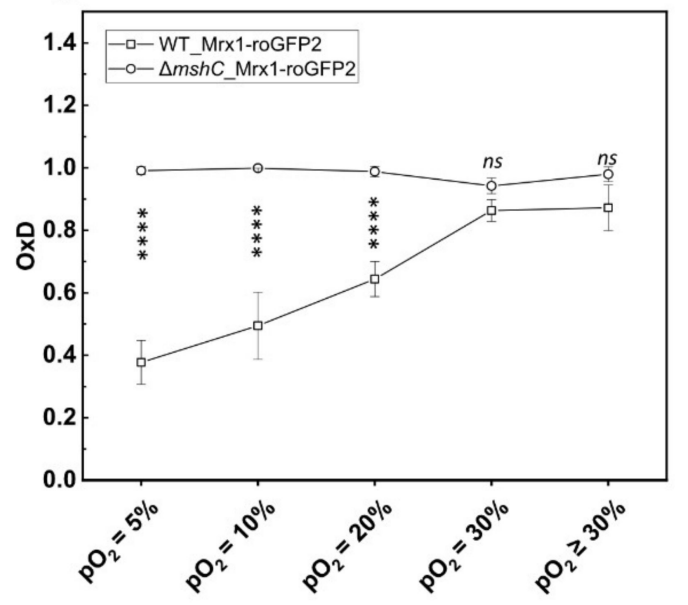

Figure 3. Growth and 380/470 nm excitation ratio of the Mrx1-roGFP2 biosensor of C. glutamicum WT_Mrx1-roGFP2 (WT_Mrx1-roGFP2) during batch cultivation in stirred bioreactors (a) and calculated oxidation degree $(\mathrm{OxD})$ at different $\mathrm{pO}_{2}$ levels during batch fermentation conducted with WT_Mrx1-roGFP2 and the MSH-deficient mutant strain C. glutamicum $\Delta m s h$ C_Mrx1-roGFP2 $\left(\triangle m s h C_{-}\right.$Mrx1-roGFP2) (b). Fermentation was performed in BIOSTAT ${ }^{\circledR}$ B bioreactors using CGXII minimal medium $\left(\mathrm{T}=30^{\circ} \mathrm{C}, \mathrm{pH}=7.0\right.$; initial glucose concentration $\left.20 \mathrm{~g} \mathrm{~L}^{-1}\right)$. OxD values at respective $\mathrm{pO}_{2}$ levels were calculated by normalizing fluorescence measurements to fully oxidized (200 mM $\mathrm{CHP}, 15 \mathrm{~min}$ incubation) and reduced (10 mM DTT, 15 min incubation) controls. Error bars indicate standard deviation of six fluorescence measurements. Significance of difference between OxD values of WT_Mrx1-roGFP2 and $\triangle m s h C \_M r x 1$-roGFP2 at different $\mathrm{pO}_{2}$ levels was determined by one-way ANOVA and Tukey's test at the 0.01 level ( ${ }^{\mathrm{ns}} p \geq 0.01$, ${ }^{* * * *} p<0.00001$ ).

\section{Discussion}

Utilization of respiratory chains for aerobic metabolism comes along with the generation of ROS [6,9]. To eliminate these toxic byproducts, aerobic organisms developed antioxidant defense mechanisms including enzymatic and non-enzymatic protection systems [12-17]. The abundant LMW thiol MSH functions to maintain the reduced state of the cytoplasm and represents the main non-enzymatic antioxidant in high-GC Gram-positive bacteria, such as the industrial platform organism C. glutamicum $[19,27,48]$. Apart from MSH, C. glutamicum encodes highly efficient enzymatic detoxification systems, such as the superoxide dismutase (SOD) [49] and methionine sulfoxide reductases (Msr) [50]; catalase (KatA) and the peroxiredoxins mycothiol peroxidase (Mpx) [15,51]; and thiol-peroxidase (Tpx) [27]. The metalloenzyme superoxide dismutase (SOD) (EC 1.15.1.1) catalyzes the dismutation of superoxide anions to $\mathrm{H}_{2} \mathrm{O}_{2}$ and oxygen. An E. coli $\operatorname{sod} A$ sodB double mutant was impaired in growth during batch cultivation when the dissolved oxygen concentration was shifted from $30 \%$ to $300 \%$ air saturation, indicating its importance in ROS detoxification during bioreactor experiments [52]. $\mathrm{H}_{2} \mathrm{O}_{2}$ is subsequently converted to $\mathrm{H}_{2} \mathrm{O}$ and $\mathrm{O}_{2}$ via the $\mathrm{H}_{2} \mathrm{O}_{2}$ scavenging systems KatA, Mpx, and Tpx in C. glutamicum [15,27]. This avoids a further conversion to the highly toxic hydroxyl radical.

In contrast to KatA, the peroxiredoxins Mpx and Tpx employ peroxidatic Cys residues for $\mathrm{H}_{2} \mathrm{O}_{2}$ detoxification, leading to $S$-mycothiolation and intramolecular disulfides of Mpx and Tpx. Regeneration or their catalytic activities requires coupling to either the Trx/TrxR or Mrx1/MSH/Mtr pathway, whereas in biochemical studies, the latter was shown to be kinetically favored under oxidative stress $[14,15,47,53]$. Moreover, the Trx/TrxR pathway is known to be employed by many biochemical systems for regeneration, and thus regarded as rate limited [14]. Thus, the Mrx1/MSH/Mtr pathway functions in de-mycothiolation under stress conditions, when $\operatorname{Tr} x / \operatorname{Tr} x \mathrm{R}$ is busy with reduction of cellular disulfides. In C. glutamicum, the kat $A$ mutant was shown to be highly sensitive towards $\mathrm{H}_{2} \mathrm{O}_{2}$ treatment, resulting in a strong oxidation of the Mrx1-roGFP2 biosensor [29]. In contrast, no sensitivity 
towards $\mathrm{H}_{2} \mathrm{O}_{2}$ exposure was observed in the $m p x$ and t $p x$ mutants, which showed similar biosensor responses under $\mathrm{H}_{2} \mathrm{O}_{2}$ stress as the wild type [29]. C. glutamicum WT was shown to be resistant towards $100 \mathrm{mM} \mathrm{H}_{2} \mathrm{O}_{2}$ and the Mrx1-roGFP2 biosensor did not respond to $10 \mathrm{mM} \mathrm{H}_{2} \mathrm{O}_{2}$ [29]. Of note, 1-5 mM $\mathrm{H}_{2} \mathrm{O}_{2}$ resulted in a maximal roGFP2 biosensor oxidation in E. coli [12]. This indicates that KatA plays a crucial role for $\mathrm{H}_{2} \mathrm{O}_{2}$ detoxification, whereas the contribution of Mpx and Tpx is neglectable.

Notably, KatA of C. glutamicum possesses a remarkably high activity and is even commercially available (Merck, CAS Number 9001-05-2). Despite the extraordinary enzymatic detoxification power of KatA, elevated oxygen concentrations during batch fermentations resulted in cell death of the MSH-deficient C. glutamicum mutant. ROS production rates are proportional to the collision frequency of oxygen and redox enzymes [9]. Consequently, the rate of ROS production inside the cell directly depends on the oxygen concentration in the extracellular environment $[7,9]$. This indicates that ROS production in bioreactor cultivations overwhelmed the remaining antioxidant systems and that MSH as an additional antioxidant is required to provide protection against oxidative stress at elevated oxygen concentrations. Consistently, a strong oxidative response of the Mrx1-roGFP2 biosensor was observed when C. glutamicum WT was exposed to oxygen concentrations that were shown to be harmful for the MSH-deficient mutant strain in bioreactors. This confirms the requirement of MSH as supporting antioxidant and, consequently, an oxidative redox shift of the 2MSH/MSSM redox couple occurring in C. glutamicum WT strains under these conditions. Thus, the increased MSH synthesis or the overexpression of Mtr for MSH recycling could be beneficial to improve the intracellular production process, providing a promising strategy for the development of highly robust industrial production strains.

MSH has multiple antioxidant functions by scavenging free radicals either directly or as a cofactor for antioxidant enzymes [19]. When the oxygen concentration surpasses the air saturation, MSH becomes a crucial player to overcome oxidative stress, despite the presence of other highly efficient enzymatic antioxidant systems working independently of MSH.

Supplementary Materials: The following supplementary figures are available online at http://www.mdpi.com/ 2076-3921/9/10/969/s1, Figure S1: Spectral scan of the Mrx1-roGFP2 biosensor of crude cell extracts of C. glutamicum WT_Mrx1-roGFP2; Figure S2: Growth and oxidation ratio of the biosensor Mrx1-roGFP2 of C. glutamicum $\triangle \mathrm{mshC}$ _Mrx1-roGFP2 during batch cultivation in stirred bioreactors.

Author Contributions: Conceptualization, G.M.S. and F.S.F.H.; methodology, F.S.F.H., G.M.S., and L.C.; validation, F.S.F.H. and Q.N.T.; formal analysis, F.S.F.H. and Q.N.T.; investigation, F.S.F.H., G.M.S., and L.C.; resources, G.M.S. and H.A.; data curation, F.S.F.H., L.C., and G.M.S.; writing-original draft preparation, F.S.F.H. and G.M.S.; writing-review and editing, F.S.F.H., L.C., Q.N.T., G.M.S., and H.A.; visualization, F.S.F.H.; supervision, G.M.S.; funding acquisition, G.M.S. and H.A. All authors have read and agreed to the published version of the manuscript.

Funding: This work was partially funded by the Novo Nordisk Fonden within the framework of the Fermentationbased Biomanufacturing Initiative (FBM) (FBM-grant: NNF17SA0031362) and by the Bio Based Industries Joint Undertaking under the European Union's Horizon 2020 research and innovation program under grant agreement No 790507 to G.S.. We further acknowledge funding by the ERC Consolidator grant (GA 615585) MYCOTHIOLOME to H.A.

Acknowledgments: We would like to thank the Fermentation Core at DTU Bioengineering for excellent technical support, Vu Van Loi (FU Berlin) for support with biosensor calculations, and Reinhard Krämer (University of Cologne) for continuous support.

Conflicts of Interest: The authors declare no conflict of interest.

\section{References}

1. Eggeling, L.; Bott, M. A giant market and a powerful metabolism: L-lysine provided by Corynebacterium glutamicum. Appl. Microbiol. Biotechnol. 2015, 99, 3387-3394. [CrossRef] [PubMed]

2. Heider, S.A.E.; Wendisch, V.F. Engineering microbial cell factories: Metabolic engineering of Corynebacterium glutamicum with a focus on non-natural products. Biotechnol. J. 2015, 10, 1170-1184. [CrossRef] [PubMed]

3. Becker, J.; Wittmann, C. Industrial microorganisms: Corynebacterium glutamicum. Ind. Biotechnol. Microorg. 2017, 1, 183-220. 
4. Lange, J.; Münch, E.; Müller, J.; Busche, T.; Kalinowski, J.; Takors, R.; Blombach, B. Deciphering the adaptation of Corynebacterium glutamicum in transition from aerobiosis via microaerobiosis to anaerobiosis. Genes 2018, 9, 297. [CrossRef]

5. Briki, A.; Kaboré, K.; Olmos, E.; Bosselaar, S.; Blanchard, F.; Fick, M.; Guedon, E.; Fournier, F.; Delaunay, S. Corynebacterium glutamicum, a natural overproducer of succinic acid? Eng. Life Sci. 2020, 20, 205-215. [CrossRef]

6. Imlay, J.A. The molecular mechanisms and physiological consequences of oxidative stress: Lessons from a model bacterium. Nat. Rev. Microbiol. 2013, 11, 443-454. [CrossRef]

7. Imlay, J.A. How obligatory is anaerobiosis? Mol. Microbiol. 2008, 68, 801-804. [CrossRef]

8. Imlay, J.A. Pathways of oxidative damage. Annu. Rev. Microbiol. 2003, 57, 395-418. [CrossRef]

9. Korshunov, S.; Imlay, J.A. Detection and quantification of superoxide formed within the periplasm of Escherichia coli. J. Bacteriol. 2006, 188, 6326-6334. [CrossRef]

10. Messner, K.R.; Imlay, J.A. The identification of primary sites of superoxide and hydrogen peroxide formation in the aerobic respiratory chain and sulfite reductase complex of Escherichia coli. J. Biol. Chem. 1999, 274, 10119-10128. [CrossRef]

11. Antelmann, H.; Helmann, J.D. Thiol-based redox switches and gene regulation. Antioxid. Redox Signal. 2011, 14, 1049-1063. [CrossRef] [PubMed]

12. Van Der Heijden, J.; Vogt, S.L.; Reynolds, L.A.; Peña-Díaz, J.; Tupin, A.; Aussel, L.; Finlay, B.B. Exploring the redox balance inside Gram-negative bacteria with redox-sensitive GFP. Free Radic. Biol. Med. 2016, 91, $34-44$. [CrossRef] [PubMed]

13. Seaver, L.C.; Imlay, J.A.; Loewen, P. Alkyl hydroperoxide reductase is the primary scavenger of endogenous hydrogen peroxide in Escherichia coli. J. Bacteriol. 2001, 183, 7173-7181. [CrossRef] [PubMed]

14. Si, M.; Xu, Y.; Wang, T.; Long, M.; Ding, W.; Chen, C.; Guan, X.; Liu, Y.; Wang, Y.; Shen, X.; et al. Functional characterization of a mycothiol peroxidase in Corynebacterium glutamicum that uses both mycoredoxin and thioredoxin reducing systems in the response to oxidative stress. Biochem. J. 2015, 469, 45-57. [CrossRef]

15. Pedre, B.; Van Molle, I.; Villadangos, A.F.; Wahni, K.; Vertommen, D.; Turell, L.; Erdogan, H.; Mateos, L.M.; Messens, J. The Corynebacterium glutamicum mycothiol peroxidase is a reactive oxygen species-scavenging enzyme that shows promiscuity in thiol redox control. Mol. Microbiol. 2015, 96, 1176-1191. [CrossRef]

16. Van Laer, K.; Hamilton, C.J.; Messens, J. Low-molecular-weight thiols in thiol-disulfide exchange. Antioxid. Redox Signal. 2013, 18, 1642-1653. [CrossRef]

17. Morgunov, I.G.; Karpukhina, O.V.; Kamzolova, S.V.; Samoilenko, V.A.; Inozemtsev, A.N. Investigation of the effect of biologically active threo-Ds-isocitric acid on oxidative stress in Paramecium caudatum. Prep. Biochem. Biotechnol. 2018, 48, 1-5. [CrossRef]

18. Imber, M.; Pietrzyk-Brzezinska, A.J.; Antelmann, H. Redox regulation by reversible protein S-thiolation in Gram-positive bacteria. Redox Biol. 2019, 20, 130-145. [CrossRef]

19. Reyes, A.M.; Pedre, B.; De Armas, M.I.; Tossounian, M.A.; Radi, R.; Messens, J.; Trujillo, M. Chemistry and redox biology of mycothiol. Antioxid. Redox Signal. 2018, 28, 487-504. [CrossRef]

20. Tung, Q.N.; Linzner, N.; Van Loi, V.; Antelmann, H. Application of genetically encoded redox biosensors to measure dynamic changes in the glutathione, bacillithiol and mycothiol redox potentials in pathogenic bacteria. Free Radic. Biol. Med. 2018, 128, 84-96. [CrossRef]

21. Jothivasan, V.K.; Hamilton, C.J. Mycothiol: Synthesis, biosynthesis and biological functions of the major low molecular weight thiol in actinomycetes. Nat. Prod. Rep. 2008, 25, 1091-1117. [CrossRef] [PubMed]

22. Newton, G.L.; Buchmeier, N.; Fahey, R.C. Biosynthesis and functions of mycothiol, the unique protective thiol of Actinobacteria. Microbiol. Mol. Biol. Rev. 2008, 72, 471-494. [CrossRef]

23. Tossounian, M.A.; Pedre, B.; Wahni, K.; Erdogan, H.; Vertommen, D.; Van Molle, I.; Messens, J. Corynebacterium diphtheriae methionine sulfoxide reductase a exploits a unique mycothiol redox relay mechanism. J. Biol. Chem. 2015, 290, 11365-11375. [CrossRef]

24. Si, M.; Zhao, C.; Zhang, B.; Wei, D.; Chen, K.; Yang, X.; Xiao, H.; Shen, X. Overexpression of mycothiol disulfide reductase enhances Corynebacterium glutamicum robustness by modulating cellular redox homeostasis and antioxidant proteins under oxidative stress. Sci. Rep. 2016, 6, 1-14. [CrossRef] [PubMed]

25. Van Loi, V.; Rossius, M.; Antelmann, H. Redox regulation by reversible protein S-thiolation in bacteria. Front. Microbiol. 2015, 6, 1-22. [CrossRef] 
26. Hillion, M.; Antelmann, H. Thiol-based redox switches in prokaryotes. Biol. Chem. 2015, 396, 415-444. [CrossRef] [PubMed]

27. Chi, B.K.; Busche, T.; Van Laer, K.; Bäsell, K.; Becher, D.; Clermont, L.; Seibold, G.M.; Persicke, M.; Kalinowski, J.; Messens, J.; et al. Protein S-Mycothiolation functions as redox-switch and thiol protection mechanism in Corynebacterium glutamicum under hypochlorite stress. Antioxid. Redox Signal. 2014, 20, 589-605. [CrossRef] [PubMed]

28. Liu, Y.; Yang, X.; Yin, Y.; Lin, J.; Chen, C.; Pan, J.; Si, M.; Shen, X. Mycothiol protects Corynebacterium glutamicum against acid stress via maintaining intracellular $\mathrm{pH}$ homeostasis, scavenging ROS, and S-mycothiolating MetE. J. Gen. Appl. Microbiol. 2016, 62, 144-153. [CrossRef]

29. Tung, Q.N.; Van Loi, V.; Busche, T.; Nerlich, A.; Mieth, M.; Milse, J.; Kalinowski, J.; Hocke, A.C.; Antelmann, H. Stable integration of the Mrx1-roGFP2 biosensor to monitor dynamic changes of the mycothiol redox potential in Corynebacterium glutamicum. Redox Biol. 2019, 20. [CrossRef]

30. Turrens, J.F. Mitochondrial formation of reactive oxygen species. J. Physiol. 2003, 552, 335-344. [CrossRef]

31. Turrens, J.F.; Freeman, B.A.; Crapo, J.D. Hyperoxia increases $\mathrm{H}_{2} \mathrm{O}_{2}$ release by lung mitochondria and microsomes. Arch. Biochem. Biophys. 1982, 217, 411-421. [CrossRef]

32. Freeman, B.A.; Topolosky, M.K.; Crapo, J.D. Hyperoxia increases oxygen radical production in rat lung homogenates. Arch. Biochem. Biophys. 1982, 216, 477-484. [CrossRef]

33. Haugaard, N. Cellular mechanisms of oxygen toxicity. Physiol. Rev. 1968, 48, 311-373. [CrossRef] [PubMed]

34. Boehme, D.E.; Vincent, K.; Brown, O.R. Oxygen and toxicity inhibition of amino acid biosynthesis. Nature 1976, 262, 418-420. [CrossRef]

35. Gregory, E.M.; Fridovich, I. Oxygen toxicity and the superoxide dismutase. J. Bacteriol. 1973, 114, $1193-1197$. [CrossRef]

36. Käß, F.; Hariskos, I.; Michel, A.; Brandt, H.J.; Spann, R.; Junne, S.; Wiechert, W.; Neubauer, P.; Oldiges, M. Assessment of robustness against dissolved oxygen/substrate oscillations for Corynebacterium glutamicum DM1933 in two-compartment bioreactor. Bioprocess Biosyst. Eng. 2014, 37, 1151-1162. [CrossRef]

37. Abe, S.; Takayama, K.I.; Kinoshita, S. Taxonomical studies on glutamic acid-producing bacteria. J. Gen. Appl. Microbiol. 1967, 13, 279-301. [CrossRef]

38. Clermont, L.; Macha, A.; Müller, L.M.; Derya, S.M.; von Zaluskowski, P.; Eck, A.; Eikmanns, B.J.; Seibold, G.M. The $\alpha$-glucan phosphorylase MalP of Corynebacterium glutamicum is subject to transcriptional regulation and competitive inhibition by ADP-glucose. J. Bacteriol. 2015, 197, 1394-1407. [CrossRef]

39. Morgan, B.; Sobotta, M.C.; Dick, T.P. Measuring EGSH and $\mathrm{H}_{2} \mathrm{O}_{2}$ with roGFP2-based redox probes. Free Radic. Biol. Med. 2011, 51, 1943-1951. [CrossRef]

40. Van Loi, V.; Harms, M.; Müller, M.; Huyen, N.T.T.; Hamilton, C.J.; Hochgräfe, F.; Pané-Farré, J.; Antelmann, H. Real-Time imaging of the bacillithiol redox potential in the human pathogen Staphylococcus aureus using a genetically encoded bacilliredoxin-fused redox biosensor. Antioxid. Redox Signal. 2017, 26, 835-848. [CrossRef]

41. Dooley, C.T.; Dore, T.M.; Hanson, G.T.; Jackson, W.C.; Remington, S.J.; Tsien, R.Y. Imaging dynamic redox changes in mammalian cells with green fluorescent protein indicators. J. Biol. Chem. 2004, 279, 22284-22293. [CrossRef] [PubMed]

42. Krause, F.S.; Blombach, B.; Eikmanns, B.J. Metabolic engineering of Corynebacterium glutamicum for 2-Ketoisovalerate production. Appl. Environ. Microbiol. 2010, 76, 8053-8061. [CrossRef] [PubMed]

43. Roenneke, B.; Rosenfeldt, N.; Derya, S.M.; Novak, J.F.; Marin, K.; Krämer, R.; Seibold, G.M. Production of the compatible solute $\alpha$-d-glucosylglycerol by metabolically engineered Corynebacterium glutamicum. Microb. Cell Factories 2018, 17, 1-14. [CrossRef] [PubMed]

44. Graf, M.; Zieringer, J.; Haas, T.; Nieß, A.; Blombach, B.; Takors, R. Physiological response of Corynebacterium glutamicum to increasingly nutrient-rich growth conditions. Front. Microbiol. 2018, 9, 1-15. [CrossRef]

45. Xu, G.; Zha, J.; Cheng, H.; Ibrahim, M.H.A.; Yang, F.; Dalton, H.; Cao, R.; Zhu, Y.; Fang, J.; Chi, K.; et al. Engineering Corynebacterium glutamicum for the de novo biosynthesis of tailored poly- $\gamma$-glutamic acid. Metab. Eng. 2019, 56, 39-49. [CrossRef]

46. Schwarzländer, M.; Dick, T.P.; Meyer, A.J.; Morgan, B. Dissecting redox biology using fluorescent protein sensors. Antioxid. Redox Signal. 2016, 24, 680-712. [CrossRef] 
47. Van Laer, K.; Buts, L.; Foloppe, N.; Vertommen, D.; Van Belle, K.; Wahni, K.; Roos, G.; Nilsson, L.; Mateos, L.M.; Rawat, M.; et al. Mycoredoxin-1 is one of the missing links in the oxidative stress defence mechanism of Mycobacteria. Mol. Microbiol. 2012, 86, 787-804. [CrossRef]

48. Liu, Y.B.; Long, M.X.; Yin, Y.J.; Si, M.R.; Zhang, L.; Lu, Z.Q.; Wang, Y.; Shen, X.H. Physiological roles of mycothiol in detoxification and tolerance to multiple poisonous chemicals in Corynebacterium glutamicum. Arch. Microbiol. 2013, 195, 419-429. [CrossRef]

49. El Shafey, H.M.; Ghanem, S.; Merkamm, M.; Guyonvarch, A. Corynebacterium glutamicum superoxide dismutase is a manganese-strict non-cambialistic enzyme in vitro. Microbiol. Res. 2008, 163, 80-86. [CrossRef]

50. Si, M.; Feng, Y.; Chen, K.; Kang, Y.; Chen, C.; Wang, Y.; Shen, X. Functional comparison of methionine sulphoxide reductase A and B in Corynebacterium glutamicum. J. Gen. Appl. Microbiol. 2017, 63, 280-286. [CrossRef]

51. Si, M.; Zhang, L.; Chaudhry, M.T.; Ding, W.; Xu, Y.; Chen, C.; Akbar, A.; Shen, X.; Liu, S.J. Corynebacterium glutamicum methionine sulfoxide reductase a uses both mycoredoxin and thioredoxin for regeneration and oxidative stress resistance. Appl. Environ. Microbiol. 2015, 81, 2781-2796. [CrossRef] [PubMed]

52. Baez, A.; Shiloach, J. Escherichia coli avoids high dissolved oxygen stress by activation of SoxRS and manganese-superoxide dismutase. Microb. Cell Factories 2013, 12, 1-9. [CrossRef] [PubMed]

53. Si, M.; Wang, T.; Pan, J.; Lin, J.; Chen, C.; Wei, Y.; Lu, Z.; Wei, G.; Shen, X. Graded response of the multifunctional 2-cysteine peroxiredoxin, CgPrx, to increasing levels of hydrogen peroxide in Corynebacterium glutamicum. Antioxid. Redox Signal. 2017, 26, 1-14. [CrossRef] [PubMed]

(C) 2020 by the authors. Licensee MDPI, Basel, Switzerland. This article is an open access article distributed under the terms and conditions of the Creative Commons Attribution (CC BY) license (http://creativecommons.org/licenses/by/4.0/). 